\title{
A Meta-Analysis of Arsenic Trioxide Combined with Transcatheter Arterial Chemoembolization for Treatment of Primary Hepatic Carcinoma
}

\author{
Ling He, Qingyun Xu, Liguo Chen, and Ruixue Chen \\ Department of Chinese Medicine, Medical College of Jinan University, Guangzhou, Guangdong 510632, China \\ Correspondence should be addressed to Liguo Chen; tchenly@jnu.edu.cn
}

Received 21 January 2016; Revised 26 April 2016; Accepted 17 May 2016

Academic Editor: Xiao-Yan Wen

Copyright (C) 2016 Ling He et al. This is an open access article distributed under the Creative Commons Attribution License, which permits unrestricted use, distribution, and reproduction in any medium, provided the original work is properly cited.

\begin{abstract}
Primary hepatic carcinoma (PHC) is one of the most common malignant tumours in the world. More and more research has shown that $\mathrm{As}_{2} \mathrm{O}_{3}$ combined with TACE has a good curative effect in treating PHC. The objectives of this study were to evaluate the therapeutic efficacy and safety of $\mathrm{As}_{2} \mathrm{O}_{3}$ combined with TACE in treating PHC. The CNKI, VIP, Wanfang, PubMed, and Cochrane databases were searched from their inception until December 2015. Randomized controlled trials (RCTs) comparing $\mathrm{As}_{2} \mathrm{O}_{3}$ combined with TACE versus TACE alone in treating PHC were identified. Stata SE 12.0 was used for data analysis. 17 RCTs with 1055 patients were included. Meta-analysis showed that, compared with TACE alone, $\mathrm{As}_{2} \mathrm{O}_{3}$ combined with TACE showed significant effects in improving the clinical efficacy rate $(P<0.01)$, decreasing the value of alpha-fetoprotein $(P<0.01)$, increasing the one-year survival rate $(P<0.01)$, and improving the quality of life of PHC patients $(P<0.01)$. Fifteen studies had mentioned adverse events, but no serious adverse effects were reported in any of the included trials. In conclusion, $\mathrm{As}_{2} \mathrm{O}_{3}$ combined with TACE therapy appears to be potentially effective in treating PHC and is generally safe. However, further studies with rigorous designs trials and multiregional cooperation trials are needed.
\end{abstract}

\section{Introduction}

Primary hepatic carcinoma (PHC) includes hepatocellular carcinoma (HCC) and intrahepatic bile duct carcinoma. It is a common malignancy, and its incidence and mortality rate are increasing annually, making it a serious threat to human health.

Surgical resection is the primary therapeutic approach for PHC but is limited by the hidden onset of PHC. Because of this hidden onset, lack of specificity of the early symptoms, and rapid progress, $\mathrm{PHC}$ is usually diagnosed in the middle-to-late stages, when it is past the window of operability [1]. Transcatheter hepatic arterial chemoembolization (TACE) is the first-line treatment for the patients with unresectable PHC. The survival benefit of TACE is supported by the results of a meta-analysis of clinical trials comparing TACE with other conservative treatments in patients with inoperable PHC [2]. The results showed that the median survival of patients improved following TACE
$[3,4]$. Chemotherapy regimens commonly used in clinical settings include 5-fluorouracil, antibiotics (mitomycin, adriamycin, and pirarubicin), and platinum drugs (cisplatin and oxaliplatin). Patients are provided with symptomatic treatment to protect the liver and acid. When necessary, patients are treated to elevate albumin and white blood cells [4]. With the wide application of TACE, the drug regimen for primary hepatic embolization and chemotherapy requires diversification, and the use of traditional Chinese medicine (TCM) is increasing.

$\mathrm{As}_{2} \mathrm{O}_{3}$, an arsenic compound, is approved and listed as an antitumour drug in some countries. In the 1970s, Professor Zhang TD found that $\mathrm{As}_{2} \mathrm{O}_{3}$, the active ingredient of which is arsenic, was effective in the treatment of leukemia, and it received attention and recognition from the international medical community. A large number of animal experiments and clinical application studies in $\mathrm{As}_{2} \mathrm{O}_{3}$ for the treatment of liver cancer have been carried out by scholars. The research showed that $\mathrm{As}_{2} \mathrm{O}_{3}$ with TACE prevents recurrence and 
metastasis in treated $\mathrm{PHC}$, primarily by changing the composition of the cancer cell nuclear matrix protein, inhibiting the expression of cancer cell proliferation cell antigens, inducing cancer cell apoptosis, and inhibiting HCC development [5]. $\mathrm{As}_{2} \mathrm{O}_{3}$ with TACE is used to treat $\mathrm{PHC}$, as seen in the increasing number of clinical research reports. However, no meta-analyses, reviews, or systematic reviews have evaluated the benefits of $\mathrm{As}_{2} \mathrm{O}_{3}$ combined with TACE in the treatment of PHC. Therefore, we evaluated the therapeutic efficacy and safety of $\mathrm{As}_{2} \mathrm{O}_{3}$ combined with TACE in the treatment of $\mathrm{PHC}$ on the basis of existing clinical studies.

\section{Materials and Methods}

2.1. Search Strategy. To ensure a complete search, we carried out a comprehensive investigation of available periodical databases and limited the search from the day of inception to December 2015. We used "arsenic trioxide (or) $\mathrm{As}_{2} \mathrm{O}_{3}$," "liver cancer (or) liver neoplasms," "primary hepatic carcinoma," and "transcatheter arterial chemoembolization (or) TACE" as keywords to retrieve studies from PubMed, Cochrane, the Chinese science and technology periodical full-text database of CNKI, the Chinese medical information resources system of VIP, and the Wanfang database.

\subsection{Inclusion Criteria}

2.2.1. Types of Studies. A randomized controlled trial (RCT) of $\mathrm{As}_{2} \mathrm{O}_{3}$ combined with TACE for the treatment of PHC, including a comprehensive statistical index and complete general information, is included.

2.2.2. Object of Studies. The objects of the studies were in accordance with the standard of defined PHC. The diagnosis criteria of PHC were based on the new methods for the diagnosis and treatment of common malignant neoplasms [5]. All cases were confirmed by pathology, cytology, and/or an imaging examination diagnosis of patients with advanced PHC.

2.2.3. Interventions. The control groups were treated with TACE. The experimental groups were treated with $\mathrm{As}_{2} \mathrm{O}_{3}$ combined with TACE, excluding those treatments that were combined with other medication studies.

2.2.4. Index of Observation. The main outcome indicators included the following: (a) the therapeutic responses, categorized according to WHO criteria as follows: complete response (CR), partial response (PR), stable disease (SD), and progressive disease (the objective efficient $=$ (the cases of complete response + the partially catabatic cases)/the total number of cases multiplied by $100 \%$ ); (b) alpha-fetoprotein (AFP): the AFP drop ratio = the cases of AFP decreasing after treatment/the cases of AFP increasing before treatment; (c) survival period: 1-year survival rate $=$ the cases of survival/total in the first year; (d) the quality of life: rate of healing increase $=$ the number of cases with a Karnofsky score of 10 points or more after treatment/total number multiplied by $100 \%$; (e) adverse reactions.

2.3. Standard of Exclusion. Studies that met any of the following criteria were excluded: (a) the inclusion of cases of metastatic HCC; (b) non-RCTs studies; (c) studies that used $\mathrm{As}_{2} \mathrm{O}_{3}$ combined with other positive drugs; and (d) control groups that were given TACE and other treatments.

2.4. Screening of Included Texts. Two reviewers independently read the titles and abstracts of the potential studies. They excluded the studies that obviously did not meet the inclusion criteria. They read the full texts of studies that met the standards to determine whether they truly met the inclusion criteria. They then cross-checked the texts and discussed disagreements or suggestions for solutions.

2.5. Quality Evaluation. We used the Cochrane Handbook 5.0.1 bias risk assessment tools to evaluate the quality of each included study [6]. The assessment criteria primarily included (a) random sequence generation (selection bias), (b) allocation concealment (selection bias), (c) blinding of participants and personnel (performance bias), (d) blinding of outcome assessment (detection bias), (e) incomplete outcome data, (f) selective reporting (reporting bias), and (g) other biases.

2.6. Extraction. Two researchers developed a form based on the inclusion and exclusion criteria to extract the following: (a) general information, including author, date of publication, age, gender, procedures, index of observation, and adverse reactions, and (b) the type of literature quality evaluation, including author, date of publication, random method, hidden allocation scheme, blind method, description of last visit, and baseline.

2.7. Statistical Processing. We used Stata SE 12.0 analysis software for the statistical analyses of the selected studies. The relative risk (odds ratio (OR)) was used to analyse the statistical data, and the weighted mean difference was used to analyse the results. The effects were expressed using 95\% confidence intervals (CIs). First, we tested for heterogeneity of the included studies using the chi-square test. When studies in the group were not heterogeneous $(P \geq 0.1$, $I^{2} \leq 50 \%$, we used the fixed-effects model for the metaanalysis. When heterogeneity $\left(P<0.1, I^{2}>50 \%\right)$ was present, we analysed the reason and used sensitivity analyses to process the data before excluding studies of lower quality and evaluating the stability of the results of the meta-analysis. The studies whose heterogeneity still could not be eliminated were consolidated with a random-effects model. We used a forest map to list the results and an intention-to-treat analysis to determine whether there was attrition bias. However, if there was clear clinical heterogeneity among the studies, we did not merge them but rather performed a descriptive qualitative analysis. The funnel plot test was used to examine the existence of publication bias. 


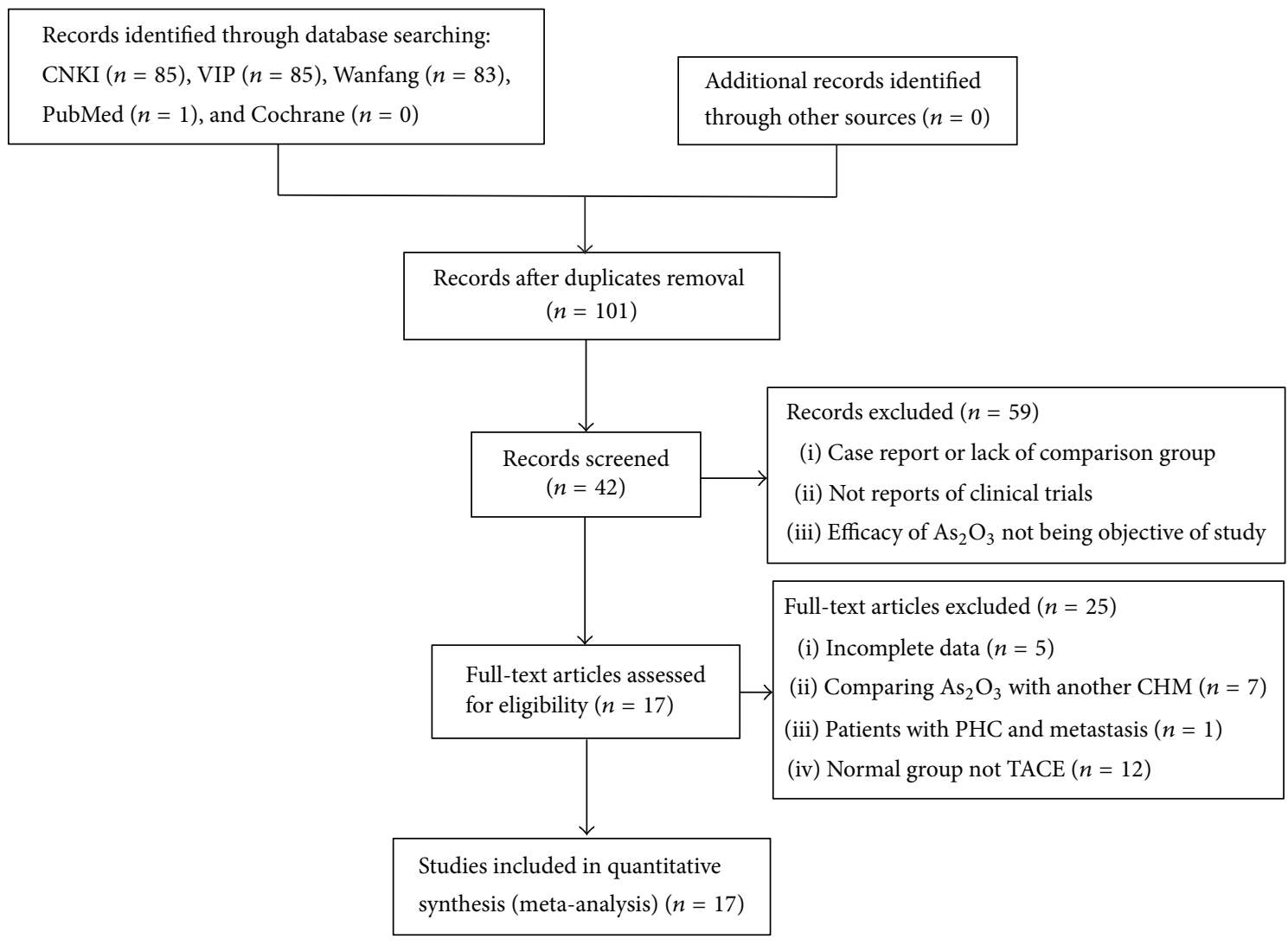

FIGURE 1: The chart of literature filtering flow.

\section{Results}

3.1. The Results of the Literature Retrieval and Screening. We initially retrieved 254 articles. The CNKI database included 85 articles, the VIP database included 85 articles, the Wanfang database included 83 articles, PubMed included 1 article, and the Cochrane database included no articles. Initially, 101 articles were excluded due to duplicate publications, which were determined by reading the title, abstract, and text. In addition, we screened those studies that did not meet the inclusion criteria or used randomized contrast groups and different measurement indexes. Finally, we identified 17 articles that met the inclusion criteria. The literature filtering flow is shown in Figure 1.

3.2. The Basic Facts of the Included Studies. There were 17 articles that met the inclusion criteria, and 1055 patients were observed, including 530 cases in the experimental group and 525 cases in the control group (see Table 1).

3.3. The Quality Assessment of Studies Included in the MetaAnalysis. We used the Cochrane Handbook 5.0.1 bias risk assessment tools to evaluate the quality of the included studies and found that 17 studies were randomized. There were 4 studies [4, 7-9] that used the random number table method to divide groups and 1 [4] that used allocation concealment. Three studies [10-12] reported the situation of loss to follow-up. The research methods in the other studies were not described (see Table 2).

3.4. Assessment for Risk of Bias. We used the Cochrane Handbook 5.0.1 bias risk assessment tools to evaluate the quality of the included studies and found that 17 studies were randomized. There were 4 studies that used the random number table method to divide the groups; and 1 study report used allocation concealment; and 3 studies reported the situation of loss to follow-up. The research methods in the other studies were not described (Figure 2).

\subsection{Analyses of Clinical Efficacy}

3.5.1. The Total Efficiency of Clinical Effect. The meta-analysis showed that the total efficiency of the clinical effect was not heterogeneous among the included studies. According to the fixed-effects model analyses, the combined OR of the treatment and control groups was 1.58 [95\% CI $(1.21,2.06)$, $P<0.01]$. The diamond was on the right side of the middle line. From test $Z$, the difference between the 2 methods in $\mathrm{PHC}$ patients was obvious (Figure 3 ).

3.5.2. Publication Bias of Clinical Effect. We analysed the efficacy rate of PHC treatment. We drew a funnel plot in which the curative effect ratio of the meta-analysis results in the test and control groups (OR) was used as the abscissa, 


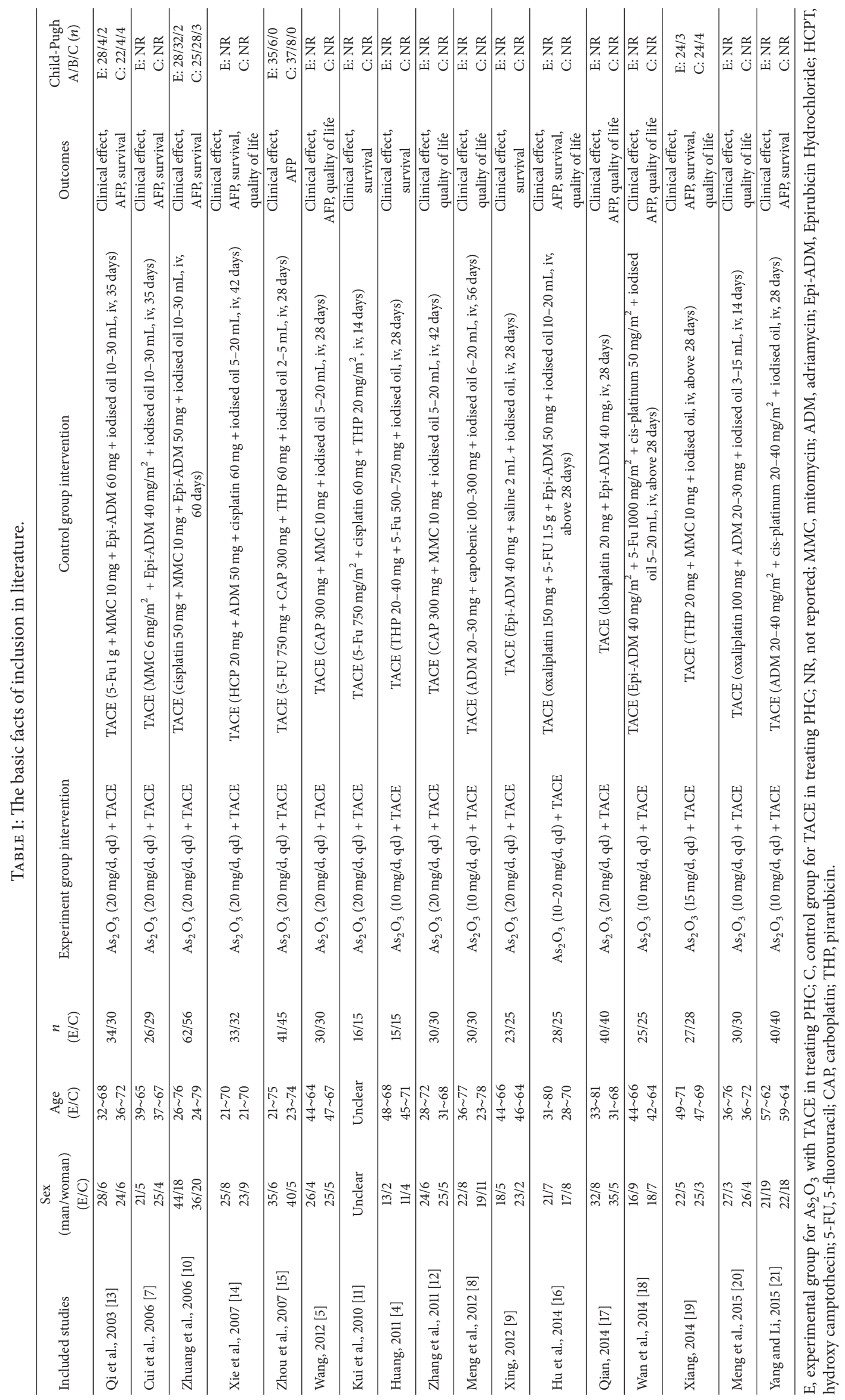


TABLE 2: The quality assessment facts of inclusion in literature.

\begin{tabular}{|c|c|c|c|c|c|c|c|}
\hline Included studies & $\mathrm{A}$ & $\mathrm{B}$ & $\mathrm{C}$ & $\mathrm{D}$ & $\mathrm{E}$ & $\mathrm{F}$ & G \\
\hline Qi et al., 2003 [13] & Unclear & Unclear & No & No & Unclear & Unclear & Unclear \\
\hline Cui et al., 2006 [7] & Unclear & Unclear & No & No & Unclear & Unclear & Unclear \\
\hline Zhuang et al., 2006 [10] & Yes & Unclear & No & No & Yes & Unclear & Unclear \\
\hline Xie et al., 2007 [14] & Unclear & Unclear & No & No & Unclear & Unclear & Unclear \\
\hline Zhou et al., 2007 [15] & Unclear & Unclear & No & No & Unclear & Unclear & Unclear \\
\hline Wang, $2012[5]$ & Unclear & Unclear & No & No & Unclear & Unclear & Unclear \\
\hline Kui et al., 2010 [11] & Unclear & Unclear & No & No & Yes & Unclear & Unclear \\
\hline Huang, $2011[4]$ & Yes & Yes & No & Yes & Unclear & Unclear & Unclear \\
\hline Zhang et al., 2011 [12] & Unclear & Unclear & No & No & Yes & Unclear & Unclear \\
\hline Meng et al., 2012 [8] & Yes & Unclear & No & No & Unclear & Unclear & Unclear \\
\hline Xing, 2012 [9] & Yes & Unclear & No & No & Unclear & Unclear & Unclear \\
\hline Hu et al., 2014 [16] & Unclear & Unclear & No & No & Unclear & Unclear & Unclear \\
\hline Qian, 2014 [17] & Unclear & Unclear & No & No & Unclear & Unclear & Unclear \\
\hline Wan et al., 2014 [18] & Unclear & Unclear & No & No & Unclear & Unclear & Unclear \\
\hline Xiang, 2014 [19] & Unclear & Unclear & No & No & Unclear & Unclear & Unclear \\
\hline Meng et al., 2015 [20] & Unclear & Unclear & No & No & Unclear & Unclear & Unclear \\
\hline Yang and Li, 2015 [21] & Unclear & Unclear & No & No & Unclear & Unclear & Unclear \\
\hline
\end{tabular}

Note: A: random sequence generation (selection bias); B: allocation concealment (selection bias); C: blinding of participants and personnel (performance bias); D: blinding of outcome assessment (detection bias); E: incomplete outcome data; F: selective reporting (reporting bias); G: other biases.

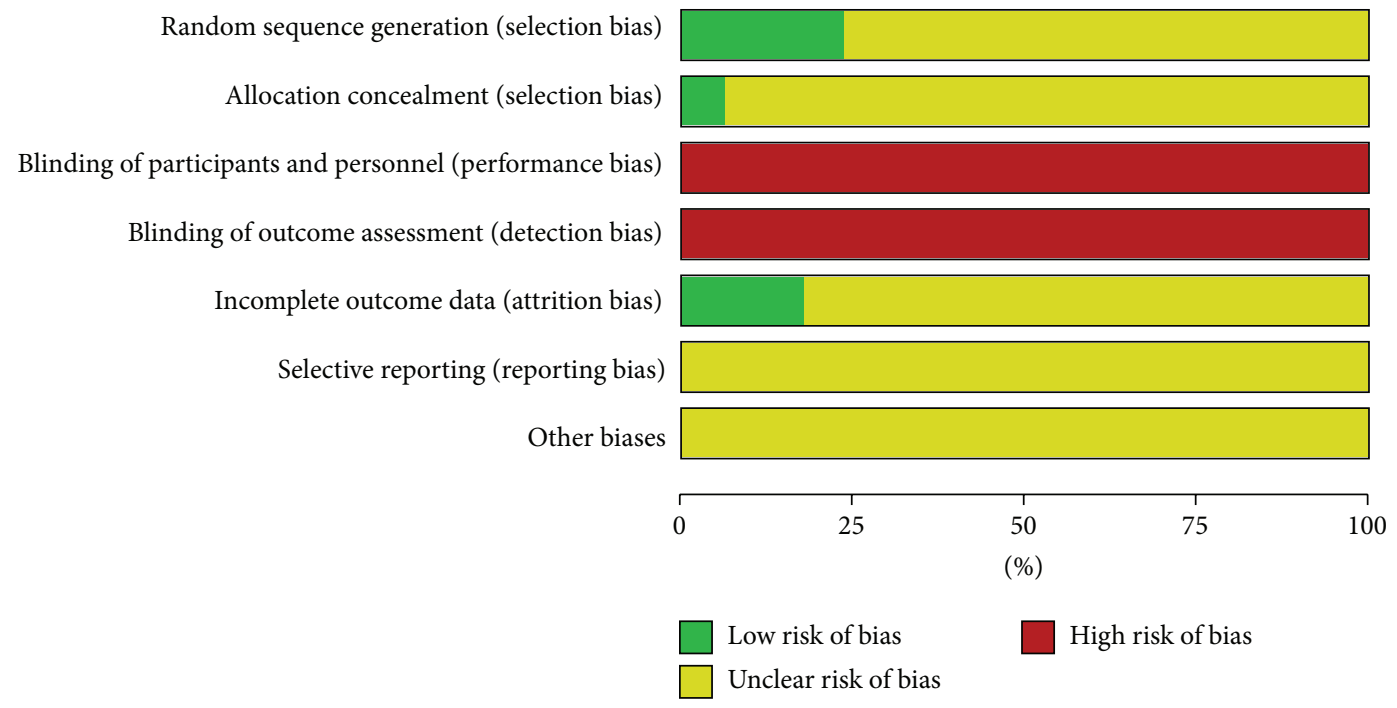

Figure 2: Bias risk assessment chart.

while $\mathrm{Se}(\log [\mathrm{or}])$ was used as the ordinate. We analysed the morphology distribution of the 17 studies using a funnel plot. The distribution was not completely symmetrical around the funnel plot, which suggested the possibility of publication bias (Figure 4).

3.5.3. The Effect of AFP. The meta-analysis showed that the AFP of the clinical effect was not heterogeneous. According to the fixed-effects model analyses, the combined OR of the treatment and control groups was 2.46 [95\% CI $(1.54,3.95)]$. The diamond was on the right side of the middle line. From test $Z$, the difference between the 2 methods in PHC patients was obvious (Figure 5).
3.5.4. Publication Bias of AFP. We analysed the efficacy rate of PHC treatment. We drew a funnel plot in which the curative effect ratio of the meta-analysis results in the test and control groups (OR) was used as the abscissa, while $\mathrm{Se}(\log [\mathrm{or}])$ was used as the ordinate. We analysed the morphology distribution using a funnel plot and found it was not completely symmetrical, which suggested the possibility of publication bias (Figure 6).

3.5.5. The Effect of One-Year Survival Rate. The meta-analysis showed that the one-year survival rate was not heterogeneous. According to the fixed-effects model analyses, the combined OR of the treatment and control groups was 3.14 


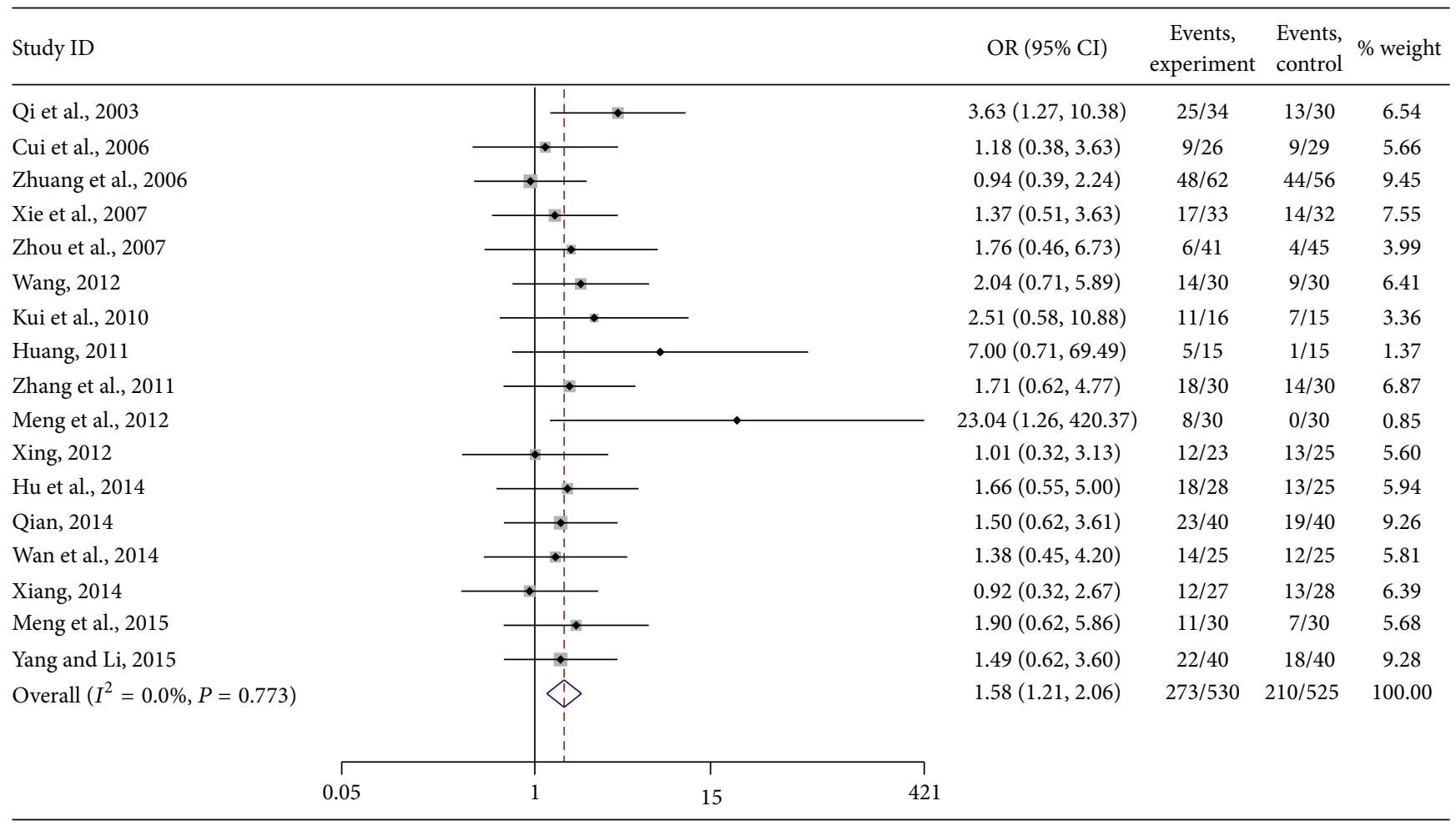

Note: weights are from random-effects analysis.

Figure 3: Meta-analysis on the total effects of $\mathrm{As}_{2} \mathrm{O}_{3}$ with TACE in treating PHC.

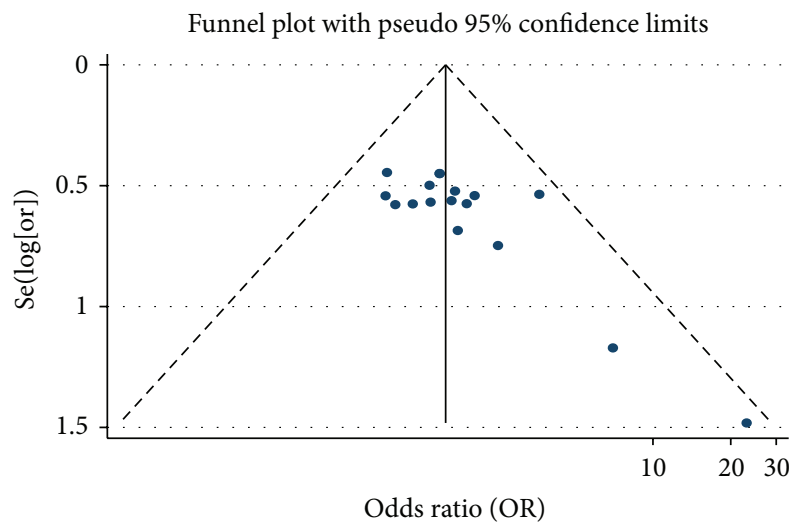

FIGURE 4: Funnel plot of the effect of $\mathrm{As}_{2} \mathrm{O}_{3}$ with TACE in treating PHC.

[95\% CI $(2.10,4.71), P<0.01]$. The diamond was on the right side of the middle line. From test $Z$, the difference between the 2 methods in PHC patients was obvious (Figure 7).

3.5.6. Publication Bias of One-Year Survival Rate. We analysed the one-year survival rate for PHC treatment. We drew a funnel plot in which the curative effect ratio of the metaanalysis results in the test and control groups (OR) was used as the abscissa, while $\mathrm{Se}(\log [$ or] $)$ was used as the ordinate. We analysed the morphology distribution using a funnel plot and found it was not completely symmetrical around the funnel plot, which suggested the possibility of publication bias (Figure 8).

3.5.7. The Effect of Life Quality. The meta-analysis showed that the quality of life effect was not heterogeneous. According to the fixed-effects model analyses, the combined OR of the treatment and control groups was 1.90 [95\% CI $(1.31,2.75)$, $P<0.01]$. The diamond was on the right side of the middle line. From test $Z$, the difference between the 2 methods in $\mathrm{PHC}$ patients was obvious (Figure 9).

3.5.8. Publication Bias of Life Quality. We analysed the life quality associated with PHC treatment. We drew a funnel plot in which the curative effect ratio of the meta-analysis results in the test and control groups (OR) was used as the abscissa, while $\mathrm{Se}(\log [\mathrm{or}])$ was used as the ordinate. We analysed the morphology distribution using a funnel plot and found that it was not completely symmetrical around the funnel plot, which suggested the possibility of publication bias (Figure 10).

3.5.9. Adverse Reactions. There were 15 studies that clearly described adverse reactions in the experimental and control groups. A variety of drug combinations may cause bloating, loss of appetite, nausea, vomiting, fever, pain, mild water sodium retention, mild oedema of the face and lower limbs, itchy skin, gastrointestinal tract reactions, haematological toxicity, and liver function damage. However, the adverse reactions were generally I II degrees, and no degree IV 


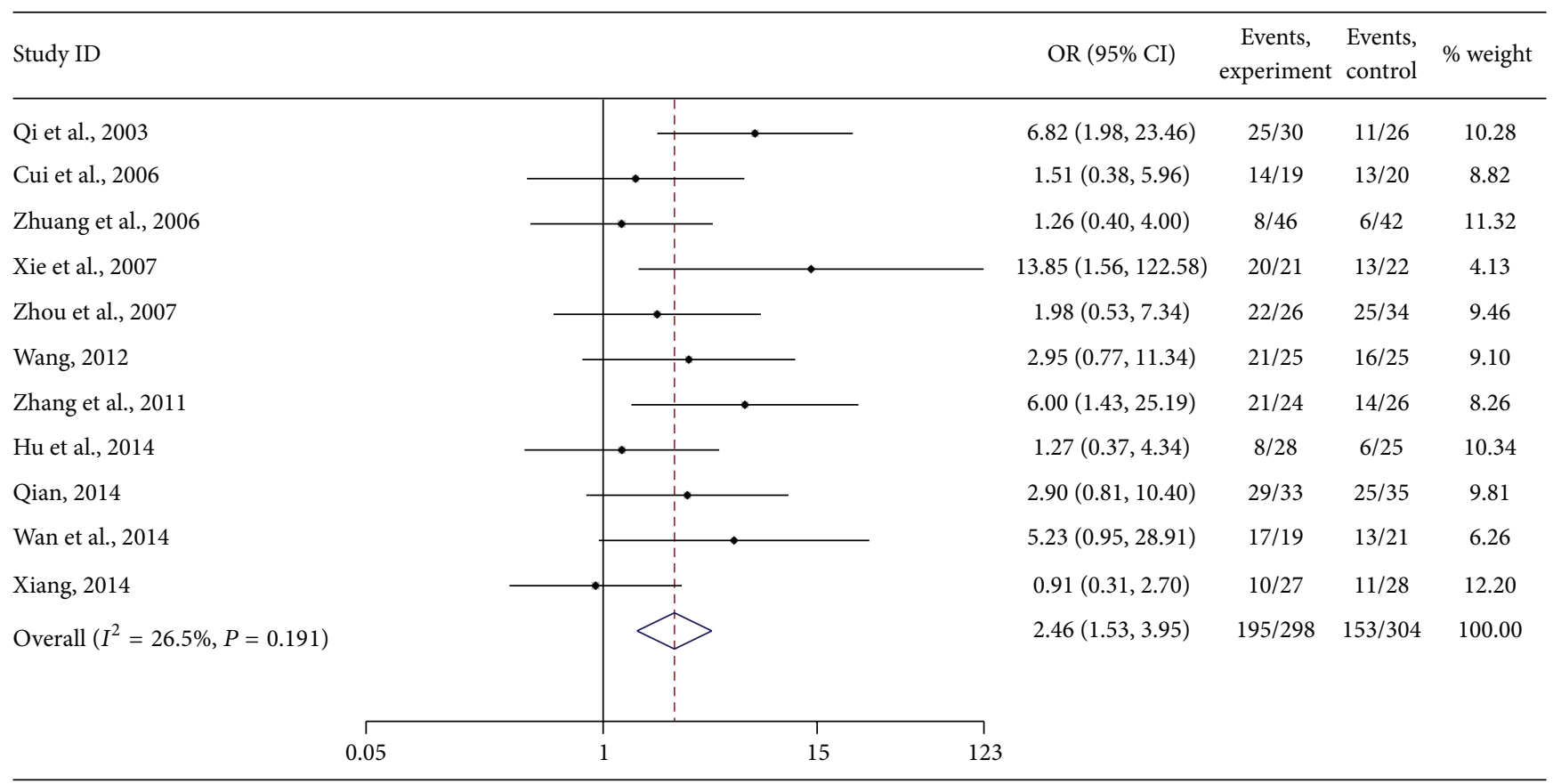

Note: weights are from random-effects analysis.

FIgure 5: Meta-analysis on the APF of $\mathrm{As}_{2} \mathrm{O}_{3}$ with TACE in treating PHC.

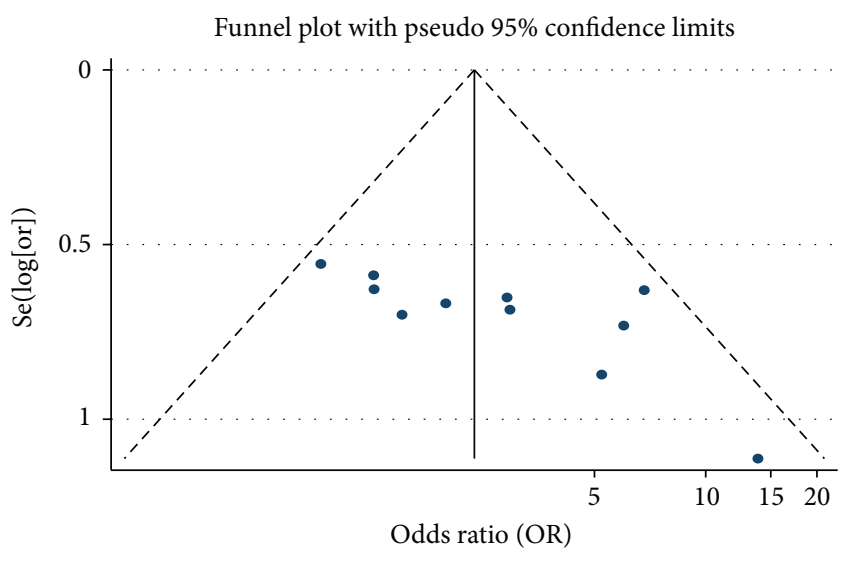

FIgURE 6: Funnel plot of APF of $\mathrm{As}_{2} \mathrm{O}_{3}$ with TACE in treating PHC.

reactions were noted. After timely and effective treatment, there were no serious complications, treatment was tolerated, and no treatment-related deaths occurred. Therefore, the TACE combination therapy is effective for the treatment of PHC.

\section{Discussion}

In the present study, a total of 17 articles were analysed to evaluate the effect of $\mathrm{As}_{2} \mathrm{O}_{3}$ with TACE in PHC patients. This meta-analysis included 1055 patients; of these, 530 patients received $\mathrm{As}_{2} \mathrm{O}_{3}$ combined with TACE therapy and 525 patients received TACE therapy. The results showed that $\mathrm{As}_{2} \mathrm{O}_{3}$ combined with TACE therapy was significantly superior to TACE alone in terms of the clinical efficacy rate $(P<0.01)$. The combined therapy decreased the AFP value $(P<0.01)$, increased the one-year survival rate $(P<$ $0.01)$, and improved the life quality of PHC patients $(P<$ $0.01)$. There were 15 studies that clearly described adverse reactions in the experimental and control groups, but no serious adverse effects were reported in any of the included trials. Therefore, the TACE combination therapy is effective for the treatment of PHC.

$\mathrm{PHC}$ is one of the most common malignant tumours in the world. It ranks fifth in incidence for malignant tumours and third in worldwide mortality. Approximately 50 million patients die from PHC each year, and its incidence is increasing. Viral hepatitis, cirrhosis, and environmental factors are thought to be causally associated with PHC. Treatment of patients with unresectable PHC is conducted with TACE [22]. TACE has advantages, including fewer adverse reactions, a tumour area with a high conventional drug concentration, an obvious curative effect, and ease of establishing collateral circulation, though it needs multiple treatments. However, the conventional repeated treatment may aggravate liver damage because it generally does not cause complete necrosis of the tumour tissue. Rather, it causes ischemia and hypoxia, which lead to increases in hypoxia-including factor and vascular endothelial growth factor expression in the tumour tissues. This treatment leads to the resistance and metastasis of the tumour, and therefore the curative effect of treatment with TACE is not ideal [17]. A rising number of $\mathrm{PHC}$ patients resort to Chinese medicine. The use of TCM is increasing. $\mathrm{As}_{2} \mathrm{O}_{3}$ is approved and listed as an antitumour drug in some 


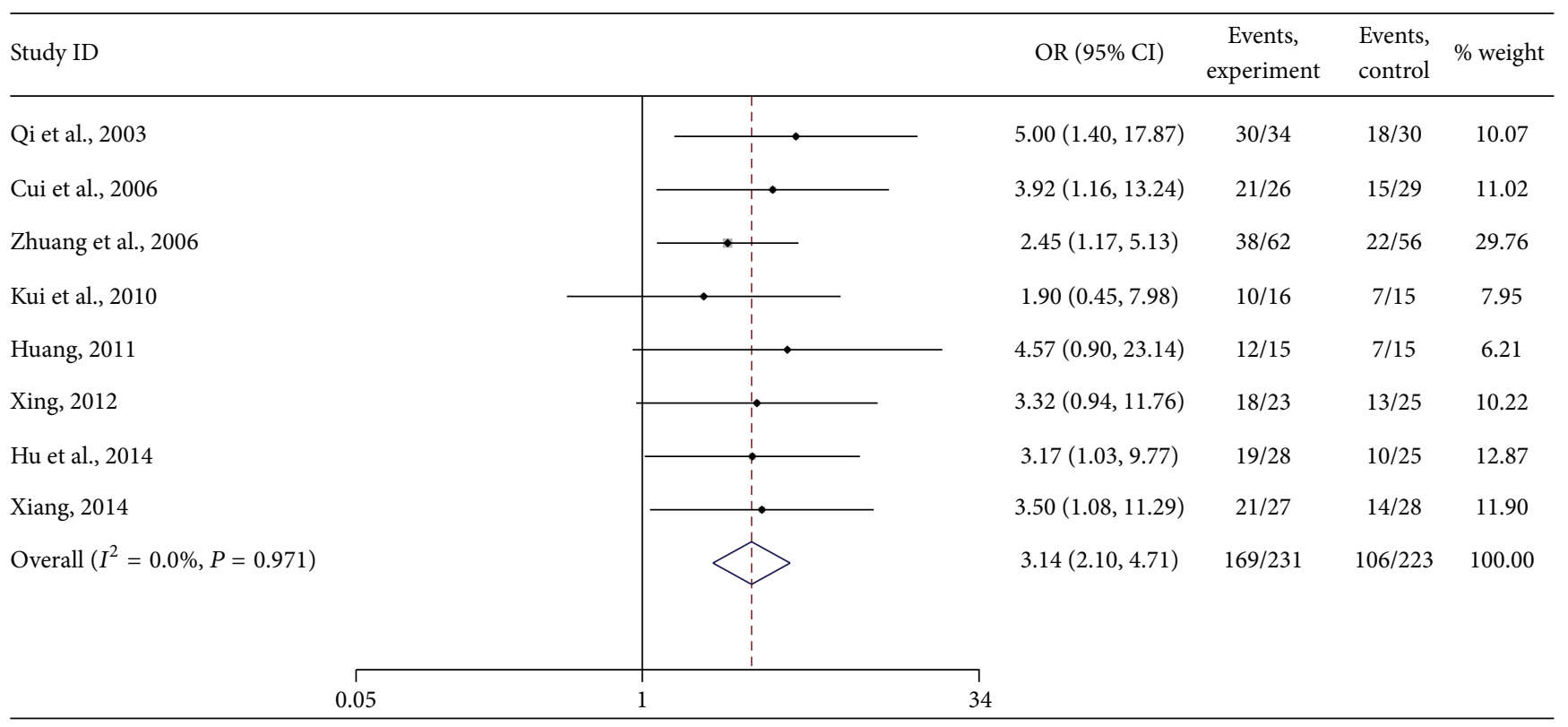

Note: weights are from random-effects analysis.

Figure 7: Meta-analysis on the one-year survival rate of $\mathrm{As}_{2} \mathrm{O}_{3}$ with TACE in treating PHC.

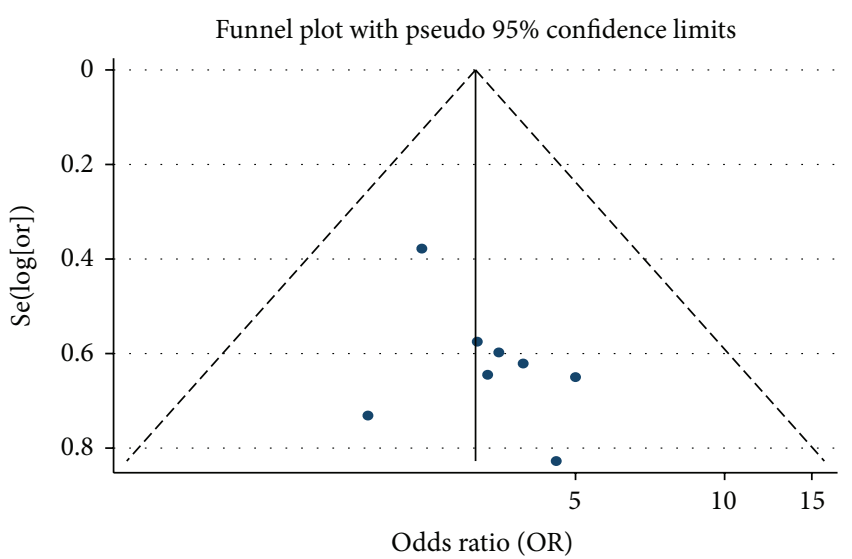

FIGURE 8: Funnel plot of one-year survival rate of $\mathrm{As}_{2} \mathrm{O}_{3}$ with TACE in treating $\mathrm{PHC}$.

countries. The inhibition of apoptosis plays an important role in the generation, development, and metastasis of malignant tumours. Apoptosis is a physiological process that is important for the preservation of homeostasis and the morphogenesis of tissues [23]. Many chemotherapeutical drugs treat malignant tumours by interfering with the pathological apoptosis regulation of tumour cells. Inducing apoptosis in tumour cells is also the operational principle of $\mathrm{As}_{2} \mathrm{O}_{3}$, an anticancer drug that has been used in traditional medicine for many centuries $[23,24]$. More and more studies show that $\mathrm{As}_{2} \mathrm{O}_{3}$ combined with TACE has a good curative effect in treating PHC.

Although this meta-analysis summarizes all available eligible studies comparing the use of $\mathrm{As}_{2} \mathrm{O}_{3}$ combined with
TACE with the use of TACE alone in treating PHC, there are still some drawbacks of this study. The evidence presented in this meta-analysis is insufficient to warrant a clinical recommendation due to the generally weak methodological quality of the included studies. The number of included RCTs was relatively small and not all studies described the method of randomization. Some significant heterogeneity may have resulted from different clinical baseline characteristics and intervention protocols among the included studies. Moreover, the values of AFP are missing which are, combined with hepatic ultrasonography, the most common markers used in clinical practice. AFP is considered to be the gold standard serum marker for the screening of patients who are at high risk of $\mathrm{PHC}$, as well as for the monitoring of treatment response [25]. The weaknesses of this paper are a result of the inherent limitations in the primary studies. First, none of the included studies in this paper were formally registered with the WHO International Clinical Trials Registry Platform. Therefore, protocols were not available to confirm that the studies were free of selective reporting. Second, all studies included in this paper used an " $A+B$ versus $B$ " design in which patients were randomized to receive either $\mathrm{As}_{2} \mathrm{O}_{3}$ combined with TACE therapy or TACE alone, and there was no rigorous control for the placebo effect. All 17 studies claimed to be RCTs, but all of them failed to give adequate and convincing information on how the random allocation was generated and concealed, which is necessary to avoid selection bias. The studies also did not mention the blinding method that was used, which could lead to performance and detection biases. No intention-to-treat analyses were mentioned, and no dropouts were reported. The 17 studies were all published in Chinese because Chinese medicine injections are used only in China. This may have produced 


\begin{tabular}{|c|c|c|c|c|c|}
\hline Study ID & & OR $(95 \% \mathrm{CI})$ & $\begin{array}{c}\text { Events, } \\
\text { experiment }\end{array}$ & $\begin{array}{l}\text { Events, } \\
\text { control }\end{array}$ & $\%$ weight \\
\hline Xie et al., 2007 & & $1.36(0.51,3.63)$ & $20 / 33$ & $17 / 32$ & 14.14 \\
\hline Wang, 2012 & & $1.97(0.70,5.54)$ & $19 / 30$ & $14 / 30$ & 12.87 \\
\hline Zhang et al., 2011 & 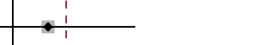 & $1.53(0.54,4.36)$ & $20 / 30$ & $17 / 30$ & 12.49 \\
\hline Meng et al., 2012 & $\rightarrow$ & $10.36(0.53,201.45)$ & $4 / 30$ & $0 / 30$ & 1.56 \\
\hline Hu et al., 2014 & & $0.92(0.31,2.72)$ & $14 / 28$ & $13 / 25$ & 11.78 \\
\hline Qian, 2014 & & $1.95(0.76,4.96)$ & $29 / 40$ & $23 / 40$ & 15.68 \\
\hline Wan et al., 2014 & & $3.86(1.18,12.61)$ & $18 / 25$ & $10 / 25$ & 9.78 \\
\hline Xiang, 2014 & & $3.60(1.18,10.95)$ & $18 / 27$ & $10 / 28$ & 11.08 \\
\hline Meng et al., 2015 & & $1.64(0.53,5.12)$ & $10 / 30$ & $7 / 30$ & 10.62 \\
\hline Overall $\left(I^{2}=0.0 \%, P=0.613\right)$ & & $1.90(1.31,2.75)$ & $152 / 273$ & $111 / 270$ & 100.00 \\
\hline 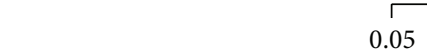 & $\begin{array}{r}1 \\
15\end{array}$ & 202 & & & \\
\hline
\end{tabular}

Note: weights are from random-effects analysis.

Figure 9: Meta-analysis on the life quality of $\mathrm{As}_{2} \mathrm{O}_{3}$ with TACE in treating PHC.

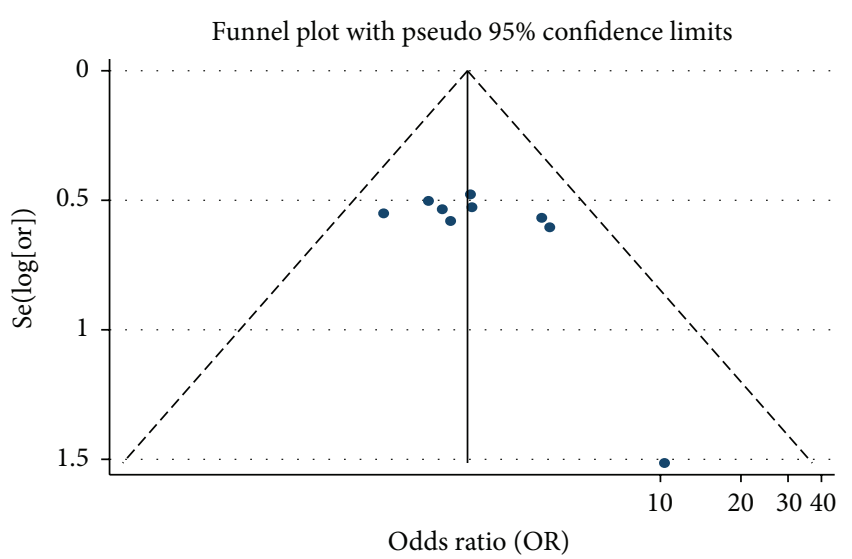

FIGURE 10: Funnel plot of the life quality of $\mathrm{As}_{2} \mathrm{O}_{3}$ with TACE in treating PHC.

a bias of publication. Most of the studies used random groups, but neither the randomization method nor the random allocation scheme was described. Therefore, there may be biases in selectivity and implementation. No use of blinding was described for the contrasting groups, which led to a high probability of selective bias. Therefore, the results and conclusions in this study should be interpreted with caution, and it will be necessary to carry out high-quality, multicentre studies with large sample sizes that are regularly reported to provide for evidence-based medicine in the future.

In conclusion, the combination of $\mathrm{As}_{2} \mathrm{O}_{3}$ with TACE was better than TACE alone in treating PHC. The combination of $\mathrm{As}_{2} \mathrm{O}_{3}$ and TACE can lower AFP, increase the one-year survival rate, improve the life quality of PHC patients, and decrease the side effects of chemotherapy. However, a variety of drug combinations may cause bloating, loss of appetite, nausea, vomiting, fever, pain, mild water sodium retention, mild oedema of the face and lower limbs, itchy skin, gastrointestinal tract reactions, haematological toxicity, and liver function damage. However, the adverse reactions were mostly I II degrees, and no degree IV reactions were reported. After timely and effective treatment, there were no serious complications, treatment was tolerated, and no treatmentrelated deaths occurred. Therefore, TACE combination therapy is effective for the treatment of PHC. Further research for application in clinical practice is needed. However, further studies with rigorous designs and large sample sizes as well as multiregional cooperation trials are needed.

\section{Competing Interests}

The authors report no competing interests in this work.

\section{Acknowledgments}

This study was supported by the National Natural Science Foundation of China (no. 81173157) and the National Natural Science Foundation of Guangdong (no. 10151063201000045).

\section{References}

[1] Y. B. Wu, "Clinical observation of Arsenic Trioxide continuous regional infusion after transcatheter hepatic arterial chemoembolization in the treatment of primary liver," Guangzhou Medical College, 2007. 
[2] J. M. Llovet and J. Bruix, "Systematic review of randomized trials for unresectable hepatocellular carcinoma: chemoembolization improves survival," Hepatology, vol. 37, no. 2, pp. 429442, 2003.

[3] J. M. Llovet and J. Bruix, "Novel advancements in the management of hepatocellular carcinoma in 2008," Journal of Hepatology, vol. 48, no. 1, pp. S20-S37, 2008.

[4] L. J. Huang, The Effects of TCM-Based Arsenic Trioxide on VEGF and Clinical Efficacy Evaluation after Transcatheter Arterial Chemoembolization in HCC, Guangzhou University of Chinese Medicine, 2011.

[5] S. M. Wang, Oleum of Brcuea Javanica and $\mathrm{As}_{2} \mathrm{O}_{3}$ Used in TACE to Treat PHC: A Clinical Study, Shandong University of Traditional Chinese Medicine, Jinan, China, 2012.

[6] J. P. T. Higgins and S. Green, Cochrane Handbook for Systematic Reviews of Interventions, Version 5.1.0, The Cochrane Collaboration, London, UK, 2011, http://handbook.cochrane.org/.

[7] S. Z. Cui, Y. B. Wu, J. K. Wang et al., "Clinical research of arsenic trioxide $\left(\mathrm{As}_{2} \mathrm{O}_{3}\right)$ continuous regional hepatic arterial infusion in the treatment of primary hepatic carcinoma," Journal of Chinese Oncology, vol. 12, no. 5, pp. 433-436, 2006.

[8] Y.-L. Meng, H.-T. Hu, H.-L. Li et al., "The clinical therapeutic effects of arsenic trioxide combined with transcatheter arterial chemoembolization in treating primary liver cancer with pulmonary metastases," Chinese Internal Medicine, vol. 51, no. 12, pp. 971-974, 2012.

[9] R. Xing, Randomized Controlled Study of Lipiodol-Arsenic Trioxide Emulsion in the Treatment of Primary Hepatic Carcinoma, China Medical University, 2012.

[10] X. L. Zhuang, X. Q. Wu, and W. Y. Wu, "Efficacy of hepatic artery infusion of arsenic trioxidein treatment of hepatocellular carcinoma," Chinese Journal of Cancer Prevention and Treatment, vol. 13, no. 14, pp. 1106-1107, 2006.

[11] G. H. Kui, J. Long, L. H. Mei et al., "Clinical study of arsenic trioxide and TACE in the treatment of middle or advanced hepatocellular carcinoma," Jiangxi Medical Journal, vol. 45, no. 6, pp. 534-536, 2010.

[12] X. B. Zhang, J. G. Xie, Z. M. Tang et al., "Clinical study of arsenic trioxide in the treatment of primary hepatic carcinoma by interventional ways," Journal of Diagnostic Imaging \& Interventional Radiology, vol. 20, no. 1, pp. 58-60, 2011.

[13] X. J. Qi, H. D. Zhao, W. B. Gao et al., "Combined percutaneous arsenic trioxide injection with transcatheter hepatic arterial chemoembolization for primary live cancer," Zhong Liu Xue Za Zhi, vol. 9, no. 3, pp. 147-149, 2003.

[14] Y. R. Xie, Y. F. Zhou, and X. L. Ying, "Clinical study of arsenic trioxide-containing regimen in the treatment of middle or advanced hepatocellular carcinoma," Zhejiang Clinical Medical Journal, vol. 9, no. 9, pp. 1161-1162, 2007.

[15] Z.-T. Zhou, X.-J. Lin, B.-K. Cui, and G.-H. Li, “Clinical study of arsenic trioxide-containing regimen in the treatment of middle or advanced hepatocellular carcinoma by TACE," Chinese Journal of Cancer Prevention and Treatment, vol. 14, no. 14, pp. 1094-1096, 2007.

[16] Q. Hu, Y. M. Wei, R. Guan, X. A. Wan, Y. H. Wang, and J. $\mathrm{Hu}$, "Clinical effect of transcatheter arteria chemoembolization combined with arsenic trioxide in the treatment of primary liver cancer," Modern Oncology, vol. 22, no. 11, pp. 2679-2681, 2014.

[17] L. K. Qian, "Clinical observation on arsenic trioxide combined with transcatheter arterial chemoembolization in treatment of patients with primary liver cancer," Chinese Medical Sciences University, 2014.
[18] X. A. Wan, Q. Zhou, and Y. M. Wei, "Efficacy of combined intraarterial and intravenous arsenic trioxideplus transcatheter arterial chemoembolization in treatment of advanced hepatocelhlar carcinoma: a report of 25 cases," Journal of Anhui University of Chinese Medicine, vol. 33, no. 6, pp. 25-27, 2014.

[19] W. Xiang, The Clinical Efficacy of Lipiodol-Arsenic Trioxide Emulsion in the Treatment of Primary Hepatic Carcinomavia TACE, Wenzhou Medical University, Wenzhou, China, 2014.

[20] Y.-L. Meng, H.-L. Li, C.-Y. Guo et al., "Arsenic trioxide combined with TACE for the treatment of multiple nodular or diffuse HCC: a clinical study," Journal of Interventional Radiology, vol. 24, no. 8, pp. 680-683, 2015.

[21] B. J. Yang and M. Li, "Inhibition effect of arsenic trioxide on liver cancer growth and metastasis," China Pharmaceuticals, vol. 24, no. 21, pp. 109-111, 2015.

[22] D. E. Ramsey and J.-F. H. Geschwind, "Chemoembolization of hepatocellular carcinoma-what to tell the skeptics: review and meta-analysis," Techniques in Vascular and Interventional Radiology, vol. 5, no. 3, pp. 122-126, 2002.

[23] M. Kito, Y. Akao, N. Ohishi, K. Yagi, and Y. Nozawa, "Arsenic trioxide-induced apoptosis and its enhancement by buthionine sulfoximine in hepatocellular carcinoma cell lines," Biochemical and Biophysical Research Communications, vol. 291, no. 4, pp. 861-867, 2002.

[24] R. Bafford, X. X. Sui, G. Wang, and M. Conte, "Angiotensin II and tumor necrosis factor-alpha upregulate survivin and Kruppel-like factor 5 in smooth muscle cells: potential relevance to vein graft hyperplasia," Surgery, vol. 140, no. 2, pp. 289-296, 2006.

[25] Y. Zhou, X. Yin, J. Ying, and B. Zhang, "Golgi protein 73 versus alpha-fetoprotein as a biomarker for hepatocellular carcinoma: a diagnostic meta-analysis," BMC Cancer, vol. 12, article 17, 2012. 


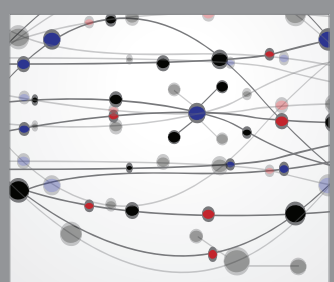

The Scientific World Journal
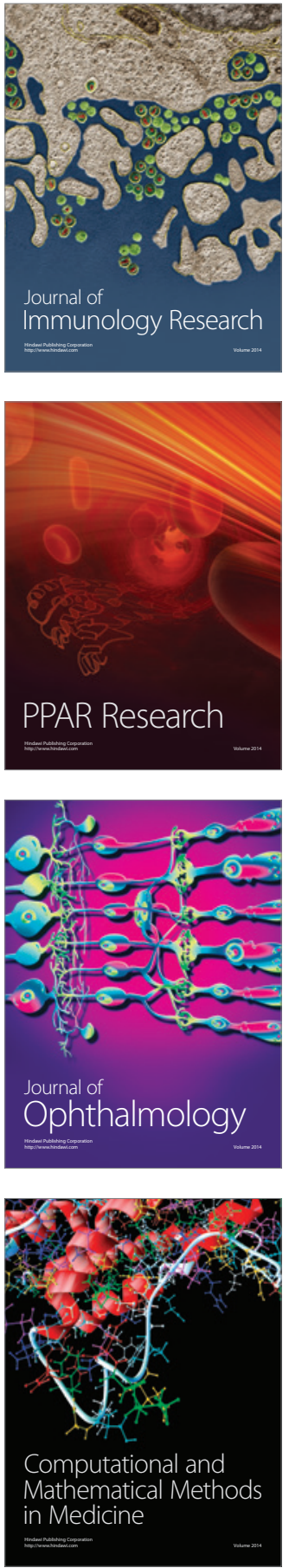

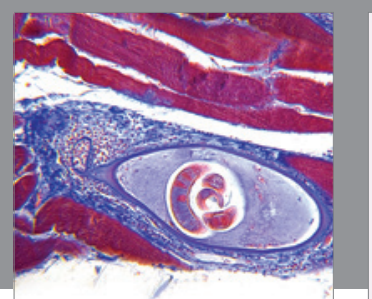

Gastroenterology Research and Practice

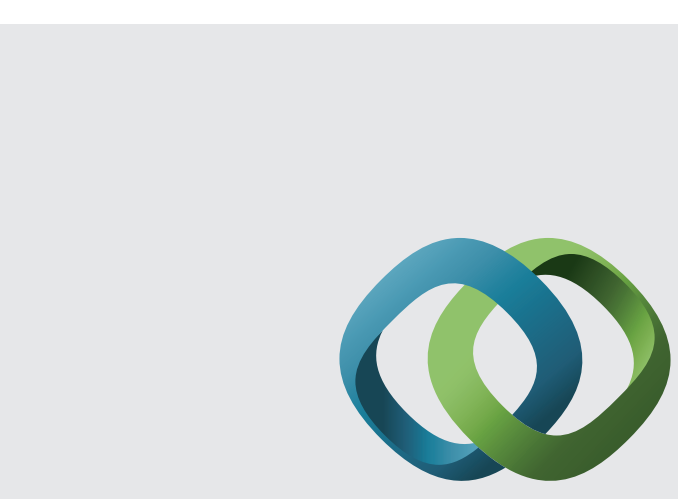

\section{Hindawi}

Submit your manuscripts at

http://www.hindawi.com
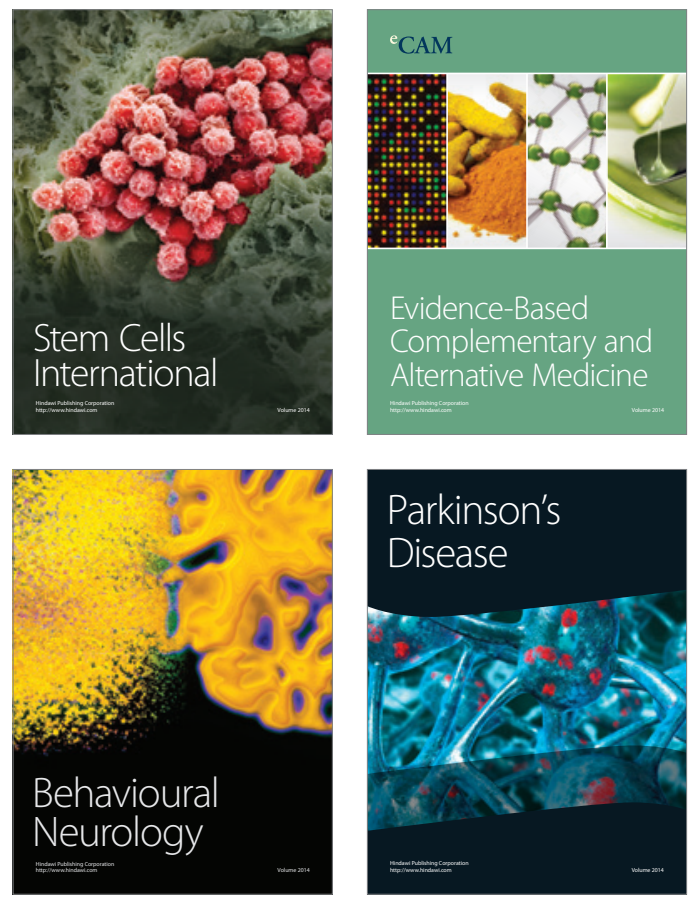
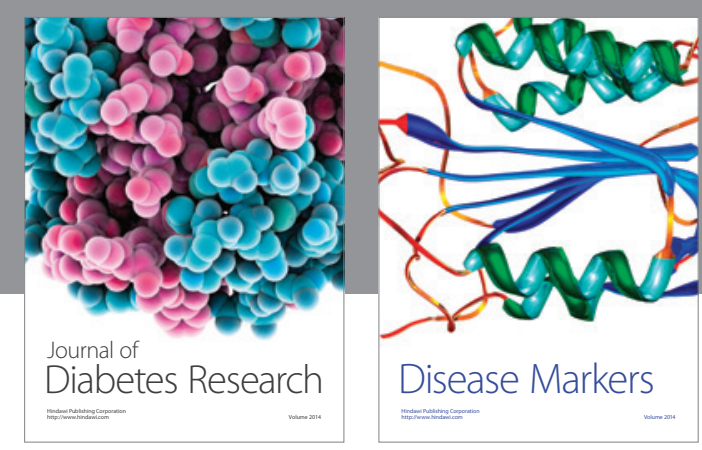

Disease Markers
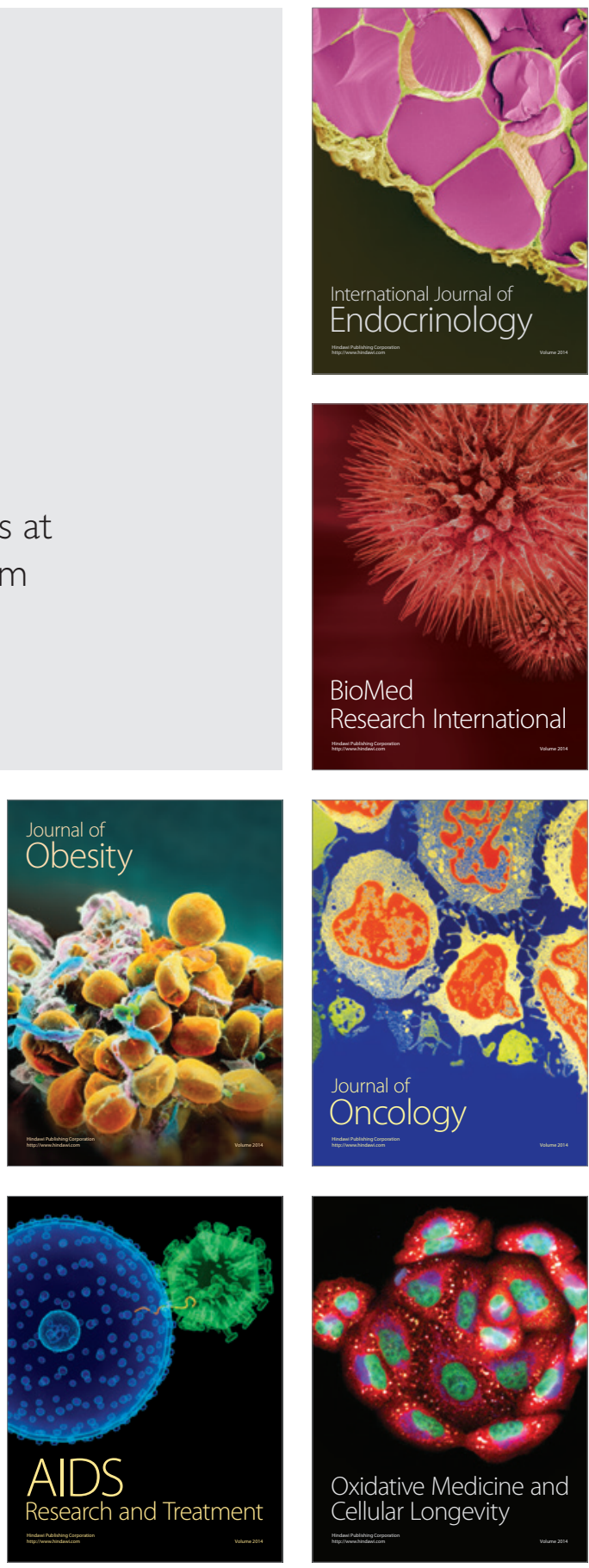